\title{
Cross sectional study of exhaled nitric oxide levels following lung transplantation
}

\author{
A J Fisher, E Gabbay, T Small, S Doig, J H Dark, P A Corris
}

\begin{abstract}
Background-The role of nitric oxide (NO) in the pathophysiology of graft dysfunction following lung transplantation remains unclear. To determine whether measurement of NO in the exhaled breath of lung transplant recipients provides useful information about graft pathology, a cross sectional study was performed on a cohort of recipients as they attended for review.

Methods-One hundred and four lung transplant recipients and 55 healthy nonsmoking controls were included in the study. Each subject performed three consecutive single breath NO manoeuvres. In recipients NO levels were compared according to current clinical status, presence of any graft pathology, type of lung transplant procedure, indication for transplantation, and current level of immunosuppression.
\end{abstract}

Results-Mean (SE) exhaled NO levels were $6.5(0.61) \mathrm{ppb}$ in the control group, $5.3(0.46)$ in clinically well recipients, 10.3 (1.4) in those with lymphocytic bronchiolitis, 10.5 (1.0) in recipients with infection, and $2.5(0.6)$ in those with acute vascular rejection. There was no significant difference in NO levels between the control group and lung transplant recipients as a whole (mean difference 0.29 (95\% CI -1.17 to 1.75$), p=0.7)$. Levels were increased significantly in the presence of lymphocytic bronchiolitis $(4.98$ (95\% CI

Department of Respiratory Medicine A J Fisher E Gabbay

T Small

S Doig

P A Corris

Department of Cardiopulmonary

Transplantation

E Gabbay

J H Dark

P A Corris

Freeman Hospital, Newcastle upon Tyne NE7 7DN, UK

Correspondence to: Dr P A Corris.

Received 24 September 1997 Returned to authors 2 December 1997

Revised version received

30 January 1998

Accepted for publication

30 January 1998 haled NO measurements may have a role as a marker of pulmonary allograft dysfunction.

(Thorax 1998;53:454-458)

Keywords: exhaled nitric oxide; lung transplantation; obliterative bronchiolitis

Nitric oxide (NO) plays an important part in physiological regulation of the pulmonary airway epithelium and pulmonary vascular endothelium. ${ }^{1}$ In addition, the regulation of NO production is implicated in the pathophysiology of airways disease. ${ }^{2}$ Upregulation of the inducible form of NO synthase (iNOS) is associated with the increased production and prolonged release of $\mathrm{NO}$, which has a role in manipulating inflammatory responses occurring in the airway. $^{2}$ The concentration of NO in exhaled breath can be measured and is increased in diseases associated with airway inflammation such as asthma ${ }^{34}$ and bronchiectasis. ${ }^{5}$

Lung transplantation now provides a realistic opportunity for prolonged survival and a better quality of life in selected patients with end-stage pulmonary disease. ${ }^{6}$ Following lung transplantation, recipients are at risk of acute deteriorations in graft function. The most common explanations for such deteriorations are acute rejection in the form of an acute vascular rejection or a bronchial and bronchiolar lymphocytic inflammation; opportunistic infections also cause considerable graft morbidity. Irreversible graft dysfunction due to the development of obliterative bronchiolitis is a serious complication. It is believed to be a manifestation of chronic rejection ${ }^{7}$ and is characterised by initially inflammatory and subsequently proliferative phases. ${ }^{8}$ It affects up to $50 \%$ of long term survivors ${ }^{9}$ and is the commonest cause of late graft failure. Obliterative bronchiolitis may be difficult to diagnose until well established. ${ }^{10}$ A clinical grading system, the bronchiolitis obliterans syndrome (BOS) grade, is used to classify recipients with obliterative bronchiolitis into grades 1,2 or 3 , depending on the severity of their obstructive ventilatory defect. ${ }^{11}$

We have previously shown that measurements of exhaled NO in a single breath are reproducible, reflect levels of NO found in the lower airways, and show little day to day variability when subjects are clinically stable. ${ }^{12}$ However, the role of exhaled NO measurements in the pathophysiology of graft dysfunction following lung transplantation remains unclear. The aims of this study were, firstly, to determine whether exhaled NO measurements in lung transplant recipients are affected by the 
type of lung transplant procedure performed, the indication for transplantation, or the recipient's current level of immunosuppression, and, secondly, we wished to establish whether exhaled NO levels in an individual provide useful information regarding the presence of inflammatory pathology in the graft. A cross sectional study on a cohort of lung transplant recipients was performed, comparing the concentration of exhaled NO in clinically well recipients with that in those with different graft pathologies. Recipients who had undergone different types of lung transplant and for different indications were compared and the effect of immunosuppression on exhaled NO was determined.

\section{Methods}

PATIENT GROUPS

Between June 1987 and May 1997 the Freeman Hospital Cardiopulmonary Transplant Unit performed 216 lung transplants in 212 recipients, 118 of whom were alive at the time of writing. The first visits of the 104 recipients (45 men) who attended for review between December 1996 and August 1997 represent the study group. The recipients had undergone different lung transplant operations: 42 single lung transplantations, 38 bilateral sequential lung transplantations, and 24 heart-lung transplantations. The indications for transplantation fell into four categories: 22 patients had obstructive lung disease, 22 interstitial lung disease, 38 suppurative lung disease, and 22 pulmonary vascular disease. Transplant recipients were receiving standard immunosuppression comprising a combination of oral prednisolone, azathioprine, and cyclosporin. Fifty five healthy, non-smoking controls ( 35 men) without a history of asthma and/or wheeze were taken from hospital staff. To avoid any effects of respiratory infections on NO levels, control subjects were free of upper or lower respiratory symptoms on the day of the test. They were asked to report any symptoms which developed within a week of the test. The study was approved by the regional ethics committee.

The recipients with graft dysfunction were divided according to their concurrent diagnoses. Recipients who presented with new symptoms or a deterioration in lung function had their diagnosis established within 24 hours of measurement of exhaled NO levels. In recipients whose pathology was identified at routine surveillance bronchoscopy, the exhaled NO level was also measured within 24 hours of their bronchoscopy. Recipients with a preexisting, well established clinical diagnosis of bronchiolitis obliterans syndrome (BOS) did not undergo a confirmational invasive assessment close to the time of NO measurement unless suspicion of other pathology was high.

The presence of infection was demonstrated by a clinically relevant culture positive throat swab or bronchoalveolar lavage culture and other concurrent diagnoses were excluded by lung biopsy when appropriate. Acute vascular rejection and lymphocytic bronchiolitis were diagnosed by a histopathologist on transbron- chial lung biopsy specimens in the presence of a sterile lavage culture. Obliterative bronchiolitis was diagnosed by characteristic obstructive changes in lung function together with evidence from transbronchial biopsy specimens and thoracic CT scans. The obliterative bronchiolitis group were further subdivided by their BOS grade into those with BOS grade 1 and those with BOS grades 2 or 3 .

NITRIC OXIDE ANALYSER

Exhaled NO was measured using a rapid highly sensitive chemiluminescence analyser (LR2000, version 2.2; Logan Research, Rochester, UK) with a resolution of 0.3 parts per billion ( $\mathrm{ppb}$ ) of $\mathrm{NO}$ and response time $(0-95 \%$ rise time) of 0.4 seconds. The analyser also measured $\mathrm{CO}_{2}$ (resolution $0.1 \% \mathrm{CO}_{2}$, response time $0.2 \mathrm{~s}$ ) by single beam infrared absorption, mouth pressure, exhaled air flow, and exhaled volume. The sampling rate was $250 \mathrm{ml} / \mathrm{min}$ for all measurements. At this flow rate the delay time was 1.4 seconds for the $\mathrm{CO}_{2}$ analyser and 1.8 seconds for the NO analyser. The analyser was calibrated daily using medical grade $\mathrm{NO}$ at a concentration of $106 \mathrm{ppb}$ in nitrogen (BOC special gases; Surrey Research Park, Guildford, $\mathrm{UK}$ ) and certified $7.9 \% \mathrm{CO}_{2}$ (Cryoservice; Worcester, UK). Mouth pressure and flow were calibrated using a water manometer and timeter (SLE limited series RT-200; Surrey, UK), respectively. Equipment was maintained in accordance with the recently published guidelines on exhaled NO measurement. ${ }^{13}$

SINGLE BREATH NITRIC OXIDE MEASUREMENTS Ambient NO concentrations were measured daily. Following a full inspiration, seated subjects were asked to exhale slowly from total lung capacity through a narrow Teflon coated tube and recordings were made of exhaled NO in parts per billion (ppb), $\mathrm{CO}_{2}(\%)$, mouth pressure $\left(\mathrm{cm} \mathrm{H}_{2} \mathrm{O}\right)$, and exhaled volume (litres). Subjects wore a nose clip and maintained mouth pressure at $4-5 \mathrm{~cm} \mathrm{H}_{2} \mathrm{O}$ by using a biofeedback visual display. At this pressure the internal nasal route is closed off by the soft palate, thus eliminating nasal NO contamination of the exhaled gas mixture. ${ }^{14} \mathrm{~A}$ constant expiratory flow $(250 \mathrm{ml} / \mathrm{s})$ was obtained as exhaled NO concentrations are highly flow dependent. ${ }^{15}$ The procedure was repeated until three technically acceptable measurements were obtained. NO measurements were recorded at end of exhalation, this point being determined from the recorded exhaled $\mathrm{CO}_{2}$ plateau. The mean of the two closest measurements was reported in accordance with British Thoracic Society (BTS) guidelines. ${ }^{16}$

COMPLEMENTARY MEASUREMENTS

Pulmonary function testing (forced expiratory volume in one second $\left(\mathrm{FEV}_{1}\right)$ and vital capacity (VC)) was performed in accordance with standardised BTS guidelines ${ }^{16}$ in the lung transplant recipients immediately before single breath NO levels were recorded.

Bronchoscopy and bronchoalveolar lavage were performed and transbronchial biopsy specimens were taken routinely at one week, 


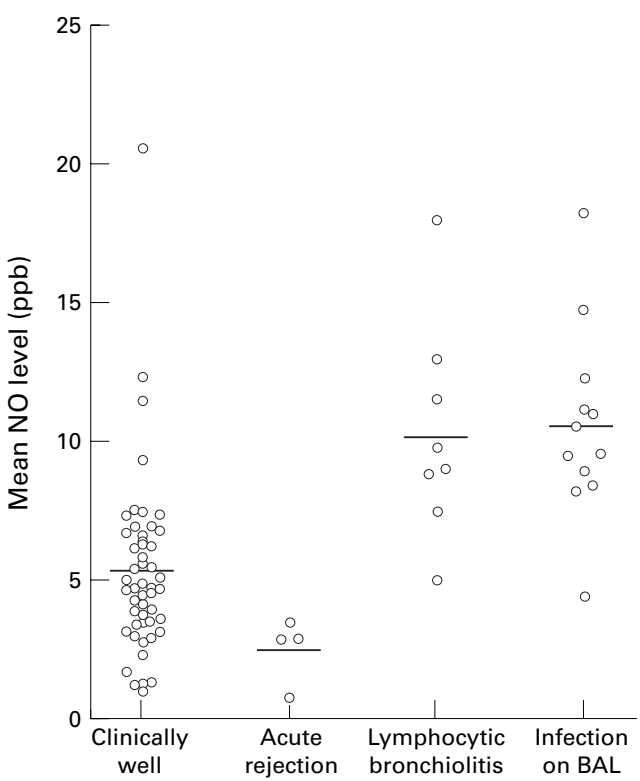

Figure 1 Mean exhaled nitric oxide (NO) levels in lung transplant recipients who were free of bronchiolitis obliterans syndrome (BOS). Comparison between clinically well recipients and disease groups: clinically well vs acute rejection, $p=N S$; clinically well vs lymphocytic bronchitis, $p=0.0002$; clinically well vs infection, $p<0.0001$.

and one, three, six and 12 months after transplantation and whenever clinically indicated. Lavage specimens were cultured for the presence of bacteria, viruses, fungi, and other potentially pathogenic organisms. Transbronchial biopsy specimens were graded for the presence of acute vascular rejection, lymphocytic bronchiolitis, and obliterative bronchiolitis in accordance with accepted guidelines. ${ }^{17}$ Acute rejection was considered significant if it was graded A2 (mild) or higher. Patients with obliterative bronchiolitis were classified according to the bronchiolitis obliterans syndrome grade as defined by the International Society of Heart and Lung Transplantation working formulation for chronic rejection. ${ }^{11}$

\section{ANALYSIS OF DATA}

Exhaled NO levels in recipients with either respiratory tract infection, acute vascular rejection, lymphocytic bronchiolitis, or obliterative bronchiolitis are expressed as mean (SE) parts per billion (ppb) and were compared with levels in recipients who were clinically well by unpaired Student's $t$ test. Differences between groups are expressed as the mean difference with the $95 \%$ confidence interval for the differences.

To determine the factors which might influence exhaled NO in lung allografts, levels were compared in recipients who had undergone the following different types of transplant: single lung, bilateral sequential lung, and heart-lung. Recipients with different transplant indications (suppurative lung disease, pulmonary vascular disease, interstitial lung disease, and obstructive lung disease) were also compared by unpaired $t$ tests. The influence on the exhaled NO level of the ambient NO concentration and the patient's current oral steroid dose and

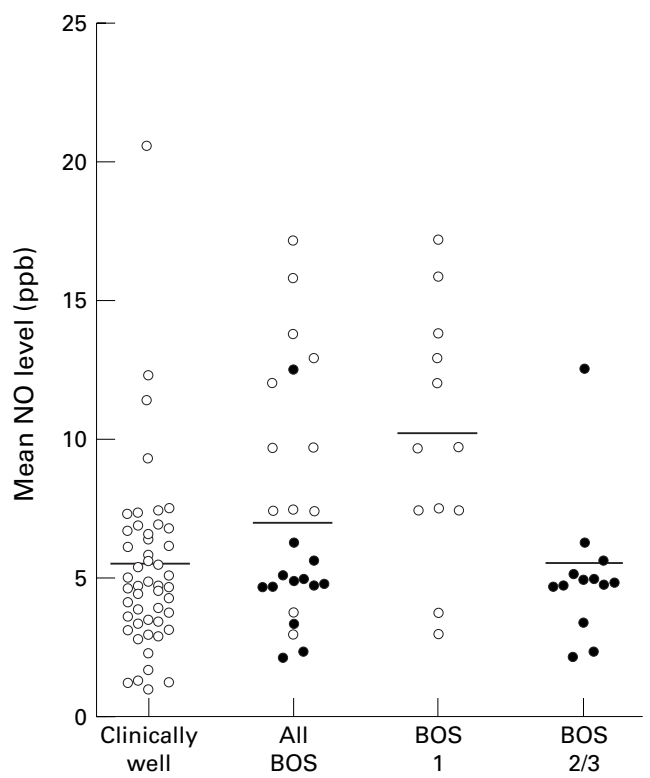

Figure 2 Mean exhaled nitric oxide (NO) levels in lung transplant recipients with bronchiolitis obliterans syndrome (BOS) compared with those who were free of BOS and clinically well: clinically well vs all BOS, $p<0.001$; clinically well vs BOS grade $1, p<0.0001$; clinically well vs BOS grades $2 / 3, p=N S$.

serum cyclosporin level was determined by univariate regression with exhaled $\mathrm{NO}$ as the dependent variable to determine the correlation coefficient. Differences were considered to have reached statistical significance if $\mathrm{p} \leqslant 0.05$.

\section{Results}

Mean (SE) single breath NO measurements were $6.8(0.42) \mathrm{ppb}$ for the lung transplant recipients as a whole and $6.5(0.61)$ for controls. There was no significant difference between the two (mean difference 0.29 (95\% $\mathrm{CI}-1.17$ to 1.75$), \mathrm{p}=0.61)$. At the time of the single breath NO measurement 50 recipients were clinically well with no previous diagnosis of obliterative bronchiolitis or other pathology on surveillance bronchoscopy. The other 54 had either pre-existing obliterative bronchiolitis or evidence of graft pathology on bronchoalveolar lavage or transbronchial biopsy. Their concurrent diagnoses were as follows: 12 had infection, four had acute vascular rejection, eight lymphocytic bronchiolitis, and 25 had obliterative bronchiolitis of which 12 were BOS grade 1 and 13 were BOS grades 2 or 3 . The remaining five had a variety of other conditions which did not fit into the classification above such as lymphoma and post-transplant lymphoproliferative disease and were not included in subsequent analysis.

Exhaled NO levels for lung transplant recipients without evidence of obliterative bronchiolitis are shown in fig 1. Mean (SE) exhaled NO levels (ppb) were $5.3(0.46)$ in clinically well recipients, $2.5(0.6)$ in those with acute vascular rejection, 10.3 (1.4) in those with lymphocytic bronchiolitis, and 10.5 (1.0) in those with infection. Compared with clinically well recipients, NO levels were significantly increased in those with lymphocytic bronchiolitis (mean difference 4.98 (95\% CI 
Table 1 Mean (SE) nitric oxide levels (ppb) in 104 lung transplant recipients according to type of transplant and underlying condition

\begin{tabular}{lcclrl}
\hline $\begin{array}{l}\text { Type of } \\
\text { transplant }\end{array}$ & No. & Mean (SE) & $\begin{array}{l}\text { Underlying } \\
\text { condition }\end{array}$ & No. & Mean (SE) \\
\hline SLTx & 42 & $7.3(0.67)$ & Obstructive & 22 & $7.3(0.91)$ \\
BSLTx & 38 & $8.0(1.23)$ & ILD & 22 & $7.1(1.00)$ \\
HLTx & 24 & $5.6(0.67)$ & Suppurative & 38 & $7.0(0.66)$ \\
Total & 104 & $6.8(0.55)$ & PVD & 22 & $5.8(0.71)$ \\
& & & Total & 104 & $6.8(0.55)$
\end{tabular}

SLTx = single lung transplant; BSLTx = bilateral sequential lung transplant; HLTx = heart-lung transplant; obstructive = obstructive lung disease; ILD = interstitial lung disease; suppurative = suppurative lung disease; PVD = pulmonary vascular disease (primary pulmonary hypertension and Eisenmenger's syndrome).

1.6 to 8.36$), p=0.0002)$ and in those with infection (mean difference 5.28 (95\% CI 2.9 to $7.56), \mathrm{p}<0.0001)$. No significant difference was demonstrated in those with acute vascular rejection compared with those who were clinically well (mean difference 2.76 (95\% CI 0.97 to 4.55$), \mathrm{p}=0.1$.

The results for recipients who had developed obliterative bronchiolitis $(n=25)$ are shown in fig 2 and compared with patients who were clinically well. For the obliterative bronchiolitis group as a whole, independent of their BOS grade, their mean exhaled NO levels were 7.4 (0.87). When this group was subdivided by their BOS grade, those with BOS grade 1 and those with BOS grades 2 or 3 had mean NO levels of 10.0 (1.3) and 5.1 (0.7), respectively. Compared with clinically well recipients, those with BOS grade 1 had significantly higher levels of NO (mean difference 4.8 (95\% CI 1.8 to $7.7), \mathrm{p}<0.0001)$, while those with BOS grade 2 or 3 had NO levels that were not significantly different from those in clinically well recipients (mean difference 0.19 (95\% CI -1.55 to 1.93 ), $\mathrm{p}=0.82)$.

There was no statistically significant correlation between exhaled NO levels and prednisolone dose $(r=-0.18)$, serum cyclosporin levels $(r=-0.22)$, or ambient NO levels $(r=$ $-0.12)$. The exhaled NO levels were not significantly different in recipients who had undergone different types of lung transplantation or in patients with different indications for transplantation (table 1).

\section{Discussion}

The discovery of measurable NO in exhaled breath has fuelled interest in the possible uses of this "new lung function test" in the diagnosis and management of pulmonary disease. ${ }^{18}$ The work of Barnes and colleagues has provided essential background information into the mechanics of exhaled $\mathrm{NO}$ measurement, the source of NO, and the association between exhaled NO levels and inflammatory diseases of the lung. ${ }^{19}$ This has led to the recent development of guidelines on the technical and clinical methodology of measuring exhaled NO levels. ${ }^{13}$

At this centre we are interested in the potential use of exhaled NO levels in a clinical role to assist in the diagnosis and management of a unique group of respiratory patients-namely, lung transplant recipients. We have shown previously, in a group of control subjects, that exhaled NO levels are repeatable and reproducible for an individual who is free of respira- tory symptoms. ${ }^{12}$ We have also shown that the exhaled NO level measured at the mouth correlates well with that measured in the lower airways at bronchoscopy. ${ }^{12}$

The results of this cross sectional analysis of 104 lung transplant recipients provides a useful insight into the potential use of exhaled NO measurements as a marker of graft dysfunction in this group of patients. The control patients used in this study were not ideal as they were not taking immunosuppression. They did, however, provide a useful group for comparison and the results did not differ from those for stable, well, lung transplant recipients. Another group such as well renal transplant recipients may have provided a more valid control group. The finding that NO levels are increased in recipients with lymphocytic bronchiolitis compared with those who are clinically well is in accordance with the results obtained in other conditions manifest by airway inflammation such as asthma. ${ }^{3}{ }^{4}$ This supports the theory that it is increased NO production in the epithelial cells due to an inflammatory upregulation of the enzyme inducible nitric oxide synthase (iNOS) which is responsible for an increase in exhaled NO levels. This theory requires further evaluation but is supported by our preliminary immunolocalisation studies (unpublished data).

The lack of a significant difference in levels of NO recorded in recipients with acute vascular rejection without evidence of lymphocytic bronchiolitis agrees with the findings of a smaller study from Toronto. ${ }^{20}$ This suggests that perivascular inflammation with a possible associated upregulation of iNOS in the endothelium is not sufficient to cause an increase in exhaled NO if the pulmonary epithelium is not involved. In the recipients with acute vascular rejection the exhaled NO level was determined before any augmentation to their immunosuppression was commenced. This suggests that the lack of difference was not due to the effect of increased immunosuppressive treatment. Several studies using animal models have shown increased levels of circulating nitrates in association with acute rejection of pulmonary and other allografts. ${ }^{21-23}$ These findings suggest that the effects of upregulated iNOS activity in the endothelium are found mainly in the vascular compartment.

Of particular interest are the raised NO levels found in patients with obliterative bronchiolitis who have early disease, BOS grade 1. This increase is lost in the more advanced stages of the disease, BOS grades 2 and 3. From our histological knowledge of obliterative bronchiolitis we recognise that the disease has a proliferative or inflammatory phase which is followed by a fibrotic and scarring stage. ${ }^{8}$ In early disease it may be that the inflammatory activity in the airways leads to increased iNOS expression and a subsequent increase in exhaled NO levels, which may be downregulated when the inflammatory phase is replaced by fibrosis. There is some evidence that iNOS is expressed in the epithelium of patients with obliterative bronchiolitis ${ }^{24}$ but this too requires further evaluation with immunolocalisation 
techniques in patients with different BOS grades.

Untreated lymphocytic bronchiolitis is believed to predispose to the development of obliterative bronchiolitis. ${ }^{25}$ The early detection of lymphocytic bronchiolitis and its adequate treatment may therefore prevent the progression to irreversible airway damage in the form of obliterative bronchiolitis. The results of this study suggest that exhaled NO measurements may have a role in the identification of airway inflammation causing graft dysfunction while it is still reversible. Further longitudinal studies are required to answer some unresolved questions such as whether a change in serial exhaled NO measurements in an individual recipient can predict the subsequent development of obliterative bronchiolitis, and whether exhaled NO levels can be used as a surrogate marker of airway inflammation in the graft to indicate response to treatment. Our further work will aim to determine the role of serial exhaled NO levels in the clinical management of lung transplant recipients.

This research was funded by the British Lung Foundation.

1 Singh S, Evans TW. Nitric oxide, the biological mediator of the decade: fact or fiction? Eur Respir f 1997;10:699-707. 2 Barnes PJ. Nitric oxide and airways disease. Ann Med 1995; 27:91-7.

3 Kharitonov SA, Yates D, Robbins RA, et al. Increased nitric oxide in exhaled air of asthmatic patients. Lancet 1994;343: 133-5.

4 Persson MG, Zetterstrom O, Agrenius V, et al. Single breath nitric oxide measurements in asthmatic patients and smoknitric oxide measurements in

5 Kharitonov SA, Wells AU, O'Connor BJ, et al. Elevated levels of exhaled nitric oxide in bronchiectasis. Am $\mathcal{F}$ Respir Crit Care Med 1995;151:1889-93.

6 Theodore J, Lewiston N. Lung transplantation comes of age. N Engl f Med 1990;322:772-4.

7 Maurer JR, Morrison D, Winton TL, et al. Late pulmonary complications of isolated lung tansplantation. Transplant Proc 1991;23:1224-5.

8 Tazelaar H, Yousem S. The pathology of combined heart-lung transplantation: an autopsy study. Hum Patho 1988;19:1403-16.
9 Tamm M, Sharples L, Dennis C, et al. Obliterative bronchiolitis in 120 consecutive heart-lung transplants. Am Rev Respir Dis 1993;147:197A.

10 Valentine VG, Robbins RC, Berry GJ, et al. Actuarial survival of heart-lung and bilateral sequential lung transplant recipients with obliterative bronchiolitis. F Heart Lung Transplant 1996;15:371-83.

11 Cooper J, Billingham M, Egan T, et al. A working formulation for the standardization of nomenclature and clinical staging of chronic dysfunction in lung allografts. $\mathcal{F}$ Heart Lung Transplant 1993;12:713-6.

12 Gabbay E, Fisher AJ, Small T, et al. Exhaled single breath nitric oxide measurements are reproducible, repeatable, and reflect levels of nitric oxide found in the lower airways. Eur Respir 71998 (in press).

13 Kharitonov SA, Alving K, Barnes PJ. Exhaled and nasal nitric oxide measurements: recommendations. ERS Task Force Report. Eur Respir f 1997;10:1683-93.

14 Kharitonov SA, Barnes PJ. There is no nasal contribution to exhaled nitric oxide during exhalation against resistance or during breath-hold. Thorax 1997;52;540-4.

15 Silkoff P, McLean P, Slutsky A, et al. Effect of pressure and flow on measurement of exhaled and nasal nitric oxide. $A m$ 7 Respir Crit Care Med 1997;155:260-7.

16 BTS/ARTP Liaison Committee. Guidelines for the measurement of respiratory function. Recommendations of the British Thoracic Society and the Association of Respiratory Technicians and Physiologists. Respir Med 1994;88: 165-94.

17 Yousem SA, Berry GJ, Cagle PT, et al. Revision of the 1990 Working Formulation for the Classification of Pulmonary Allograft Rejection: Lung Rejection Study Group. $\mathcal{F}$ Heart Lung Transplant 1996;15:1-15.

18 Borland C, Cox Y, Higenbottam T. Measurement of exhaled nitric oxide in man. Thorax 1993;48:1160-2.

19 Barnes PJ, Kharitonov SA. Exhaled nitric oxide: a new lung function test. Thorax 1996;51:233-7.

20 Caramori M, Silkoff P, McClean P, et al. Exhaled NO (ENO) in lung transplantation: the correlation with bronchoscopy. Am f Respir Crit Care Med 1997;155:A270.

21 Wiklund L, Lewis DH, Sjoquist P-O, et al. Increased levels of circulating nitrates and impaired endothelium-mediated vasodilation suggest multiple roles of nitric oxide during acute rejection of pulmonary allografts. $\mathcal{F}$ Heart Lung Transplant 1997;16:517-23.

22 Devlin J, Palmer RJ, Gonde CE, et al. Nitric oxide generation: a predictive parameter of acute allograft rejection. Transplantation 1994;58:592-5.

23 Winlaw DS, Schyvens CG, Smythe GA, et al. Urinary nitrate excretion is a non-invasive indicator of acute cardiac allograft rejection and nitric oxide production in the rat. Transplantation 1994;58:1031-6.

24 Giaid A, Corris PA, Chikhani N, et al. Expression of nitric oxide synthase in lung transplant recipients with bronchiolitis obliterans. Eur Respir f 1995;8:550S

25 Yousem SA. Lymphocytic bronchitis/bronchiolitis in lung allograft recipients. Am f Surg Pathol 1993;17:491-6. 